\title{
Chemodynamical Simulations of Galaxies and the Universe
}

\author{
Chiaki Kobayashi* \\ Research School of Astronomy and Astrophysics \\ The Australian National University \\ E-mail: chiaki@mso.anu.edu.au
}

We simulate the formation and evolution of galaxies with a hydrodynamical model including star fromation, supernova feedback, and chemical enrichment. From the comparison with observed elemental abundances, we study the evolution of galaxies and the origin of heavy elements (Galactic Archaeology).

A large fraction of hypernovae is required from the observed abundance ratios in the Milky Way Galaxy (namely, $[\mathrm{Zn} / \mathrm{Fe}] \sim 0$ ). Our progenitor model of SNe Ia based on the single degenerate scenario with the metallicity effect is prefered from the evolution of the abundance ratios (namely, $[(\alpha, \mathrm{Mn}, \mathrm{Zn}) / \mathrm{Fe}]-[\mathrm{Fe} / \mathrm{H}]$ relations $)$.

In our simulated Milky Way-type galaxy, the kinematical and chemical properties of bulge, disk, and halo are broadly consistent with observations. $80 \%$ of the bulge stars are as old as $10 \mathrm{Gyr}$, and have high $[\alpha / \mathrm{Fe}]$, while the disk stars tend to be young and show the decreasing trend of $[\alpha / \mathrm{Fe}]$ against $[\mathrm{Fe} / \mathrm{H}] .80 \%$ of thick disk stars are older than $\sim 8 \mathrm{Gyr}$, and tend to have larger $[\alpha / \mathrm{Fe}]$ than in the thin disk. We also predict the frequency distribution in the $[\mathrm{X} / \mathrm{Fe}]-[\mathrm{Fe} / \mathrm{H}]$ diagrams from carbon to zinc, depending on the location within the galaxy.

In our cosmological simulation, the hypernova feedback play an essential role in suppressing star formation, which results in the cosmic star formation rate history peaked at $z \sim 3$ as observed. The hypernova feedback also drives galactic outflows efficiently in low mass galaxies, and these winds eject heavy elements into the intergalactic medium. The ejected baryon and metal fraction is larger for less massive galaxies, which results in the observed mass-metallicity relation of galaxies. We also predict the cosmic supernova and gamma-ray burst rate hisotries, assuming that their progenitors are massive and low-metal hypernovae.

10th Symposium on Nuclei in the Cosmos

July 27 - August 12008

Mackinac Island, Michigan, USA

*Speaker. 


\section{Introduction}

While the evolution of the dark matter is reasonably well understood, the evolution of the baryonic component is much less certain because of the complexity of the relevant physical processes, such as star formation and feedback. With the commonly employed, schematic star formation criteria alone, the predicted star formation rates are higher than what is compatible with the observed luminosity density. Thus feedback mechanisms are in general invoked to reheat gas and suppress star formation. Supernovae inject not only thermal energy but also heavy elements into the interstellar medium, which can enhance star formation. Chemical enrichment must be solved as well as energy feedback. "Feedback" is also important for solving the angular momentum problem and the missing satellite problem, and for explaining the existence of heavy elements in intracluster medium and intergalactic medium, and possibly the mass-metallicity relation of galaxies ([1], hereafter K07).

In the next decade, high-resolution multi-object spectroscopy (HERMES on AAT and WFMOS on Subaru) and space astrometry mission (GAIA) will provide kinematics and chemical abundances of a million stars in the Local Group. Since different heavy elements are produced from different supernovae with different timescales, elemental abundance ratios can provide independent information on "age". Therefore, stars in a galaxy are fossils to tell the history of the galaxy. The galactic archeology technique can be used to study the galaxy formation and evolution in general. Metallicities are measured in various objects with different galaxy mass and as a function of redshift/time. The internal structure of galaxies is observed with integral field spectrographs (e.g., the SAURON project, GMOS-IFU on Gemini, SINFONI on VLT). Chemodynamical simulations can provide predictions to understand these observations.

\section{Hypernovae}

Although the explosion mechanism of core-collapse supernovae is a debated issue, the ejected explosion energy and ${ }^{56} \mathrm{Ni}$ mass (which decayed to ${ }^{56} \mathrm{Fe}$ ) can be estimated from the observations, i.e., the light curve and spectra fitting of individual supernova. As a results, it is found that hypernovae $(\mathrm{HNe})$, which have more than ten times larger explosion energy $\left(E_{51} \gtrsim 10\right)$, produce a certain amount of iron [2].

We calculate the nucleosynthesis yields for wide ranges of metallicity $\left(Z=0-Z_{\odot}\right)$ and the explosion energy (normal $\mathrm{SNe}$ II and $\mathrm{HNe}$ ). Assuming that a half of supernovae with $\leq 20 M_{\odot}$ are $\mathrm{HNe}$, the evolution of the elemental abundance ratios from oxygen to zinc (Fig.1) are in good agreement with observations in the solar neighborhood, bulge, halo, and thick disk ([3], hereafter K06). Among the $\alpha$-elements, $\mathrm{O}, \mathrm{Mg}, \mathrm{Si}, \mathrm{S}$, and $\mathrm{Ca}$ show a plateau at $[\mathrm{Fe} / \mathrm{H}] \lesssim-1$, while $\mathrm{Ti}$ is underabundant overall. The observed decrease in the odd- $\mathrm{Z}$ elements $(\mathrm{Na}, \mathrm{Al}$, and $\mathrm{Cu})$ toward low $[\mathrm{Fe} / \mathrm{H}]$ is reproduced by the metallicity effect on nucleosynthesis. The iron-peak elements $(\mathrm{Cr}, \mathrm{Mn}, \mathrm{Co}$, and $\mathrm{Ni}$ ) are consistent with the observed mean values at $-2.5 \lesssim[\mathrm{Fe} / \mathrm{H}] \lesssim-1$, and the observed trend at the lower metallicity can be explained by the energy effect. Especially, the observed abundance of $\mathrm{Zn}([\mathrm{Zn} / \mathrm{Fe}] \sim 0)$ can be explained only by such large contribution of HNe. Since the observed $[\mathrm{Zn} / \mathrm{Fe}]$ shows a increase toward lower metallicity (Primas et al. 2000; Nisesn 


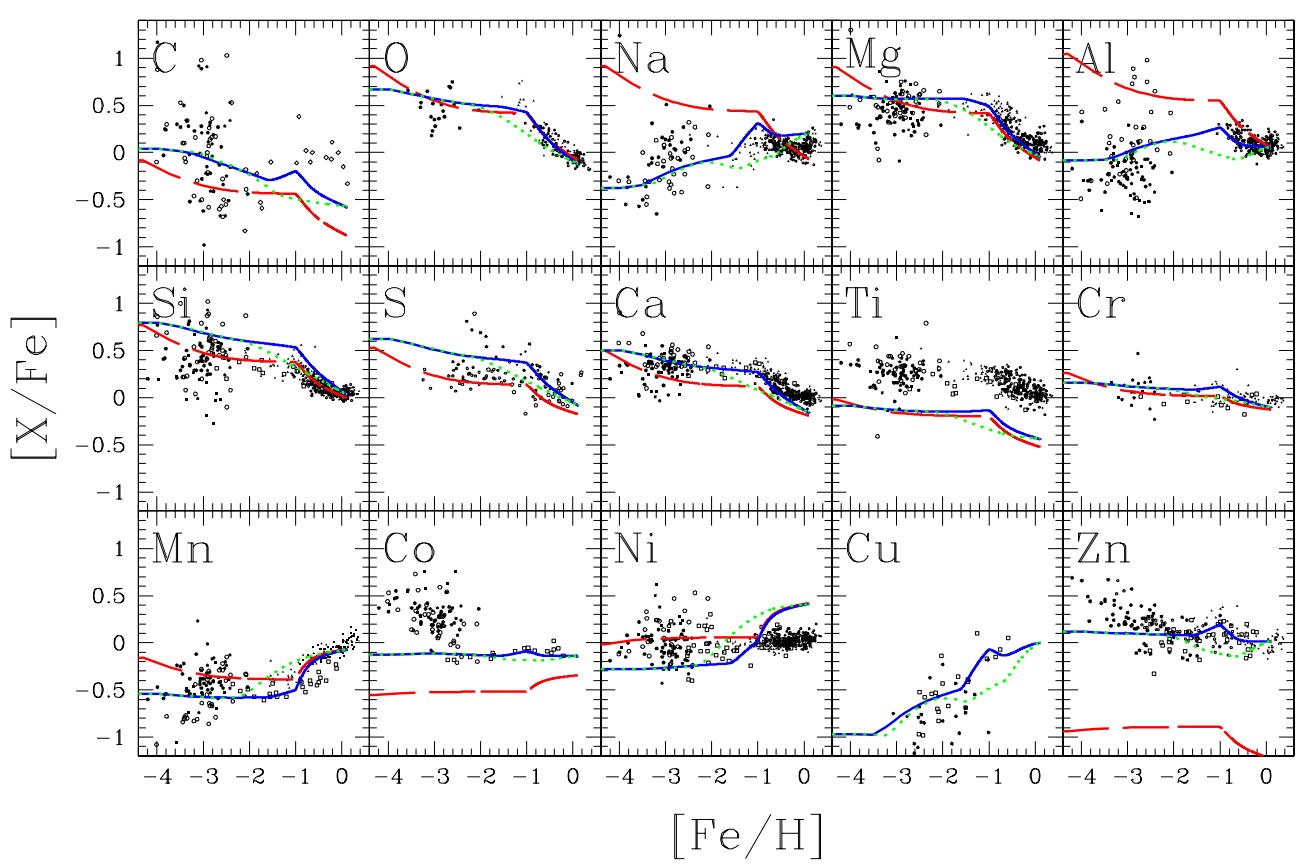

Figure 1: Evolutions of heavy element abundance ratios $[\mathrm{X} / \mathrm{Fe}]$ against $[\mathrm{Fe} / \mathrm{H}]$ for one-zone models with our new yields (solid line), and with only SNe II (dashed line), and with the double-degenerate scenario of SNe Ia (dotted line). The dots are observational data (see K06 for the references).

et al. 2007), the $\mathrm{HN}$ fraction may be larger in the earlier stage of galaxy formation. At present, the $\mathrm{HN}$ fraction can be as small as $1 \%$.

\section{Type Ia Supernovae}

The progenitors of the majority of Type Ia Supernovae (SNe Ia) are most likely the Chandrasekhar (Ch) mass white dwarfs (WDs). For the evolution of accreting $\mathrm{C}+\mathrm{O}$ WDs toward the $\mathrm{Ch}$ mass, two scenarios have been proposed: One is the double-degenerate (DD) scenario, i.e., merging of double $\mathrm{C}+\mathrm{O}$ WDs with a combined mass surpassing the $\mathrm{Ch}$ mass limit, although it has been theoretically suggested that it leads to accretion-induced collapse rather than SNe Ia. The other is our single-degenerate (SD) scenario, i.e., the WD mass grows by accretion of hydrogen-rich matter via mass transfer from a binary companion.

We construct a new model of SNe Ia, based on the SD scenario, taking account of the metallicity effect of the WD wind [4] and the mass-stripping effect [5] in the binary systems. Our model naturally predicts that the SN Ia lifetime distribution spans a range of $0.1-20 \mathrm{Gyr}$ with the double peaks at $\sim 0.1$ and $1 \mathrm{Gyr}$ reflecting the two systems of the companion stars; the main-sequence (MS)+WD and red-giants (RG) +WD systems, respectively. Because of the metallicity effect, i.e., because of the lack of winds from WDs in the binary systems, the SN Ia rate in the systems with $[\mathrm{Fe} / \mathrm{H}] \lesssim-1$, e.g., high-z spiral galaxies, is supposed to be very small.

Figure 1 shows the evolutions of heavy element abundance ratios $[\mathrm{X} / \mathrm{Fe}]$ against $[\mathrm{Fe} / \mathrm{H}]$ for one-zone models with our new yields (solid line), and with only SNe II (dashed line, Nomoto et 
al. (1997)'s yields adopted), and with the other SN Ia model (dotted line; the lifetime distribution function from the DD scenario is adopted). As the time goes, the iron abundance increases, and the abundance ratio for many elements stays constant with a plateau value at $[\mathrm{Fe} / \mathrm{H}] \lesssim-1$, which is determined only by SNe II and $\mathrm{HNe}$. From $[\mathrm{Fe} / \mathrm{H}] \sim-1$, SNe Ia start to occur producing more Fe than $\alpha$-elements, and thus $[\alpha / \mathrm{Fe}]$ decreases toward the solar abundance. The $[\mathrm{Fe} / \mathrm{H}]$ where $[\alpha / \mathrm{Fe}]$ starts to decrease depends on the SN Ia progenitor model. Our SN Ia model can give better reproduction of the $[(\alpha, \mathrm{Mn}, \mathrm{Zn}) / \mathrm{Fe}]-[\mathrm{Fe} / \mathrm{H}]$ relations than other models such as the DD scenario. With the DD scenario, the typical lifetimes of SNe Ia are $\sim 0.1 \mathrm{Gyr}$, which results in the too early decrease in $[\alpha / \mathrm{Fe}]$ at $[\mathrm{Fe} / \mathrm{H}] \sim-2$. Even with our SD model, if we do not include the metallicity effect, $[\alpha / \mathrm{Fe}]$ decreases too early because of the shortest lifetime, $\sim 0.1 \mathrm{Gyr}$, of the MS+WD systems. In other words, the presence of the young population of SNe Ia strongly favors the presence of the metallicity effect to be consistent with the chemical evolution of the solar neighborhood. Note that the star formation history and total number of SNe Ia are determined to meet the observed metallicity distribution function [6].

While the present SN Ia rate in elliptical galaxies can be reproduced with the old population of the RG+WD systems, the large SN Ia rate in radio galaxies could be explained with the young population of the MS+WD systems. We also succeed in reproducing the galactic supernova rates with their dependence on the morphological type of galaxies (Mannucci et al. 2005), and the cosmic SN Ia rate history with a peak at $z \sim 1$. At $z \gtrsim 1$, the predicted SN Ia rate decreases toward higher redshifts and SNe Ia will be observed only in the systems that have evolved with a short timescale of chemical enrichment. This suggests that the evolution effect in the supernova cosmology can be small [6].

\section{Chemodynamical Model}

The details of our chemodynamical models are described in [7], and can be summarized as follows. i) The Smoothed Particle Hydrodynamics (SPH) method is adopted with individual smoothing lengths and timesteps. For the simulations of individual galaxies, the gravity is calculated in direct summation using the special purpose computer GRAPE (GRAvity PipE). For cosmological simulations, we basically use an SPH code GADGET-2 by Springel (2005), and introduce the same models of physical processes as in the GRAPE-SPH code. ii) Radiative cooling is computed using a metallicity-dependent cooling function (Sutherland \& Dopita 1993). iii) Our star formation criteria are the same as in (Katz 1992); (1) converging flow; $(\nabla \cdot v)_{i}<0$, (2) rapid cooling; $t_{\mathrm{cool}}<t_{\mathrm{dyn}}$, and (3) Jeans unstable gas; $t_{\mathrm{dyn}}<t_{\text {sound }}$. The star formation timescale is proportional to the dynamical timescale $\left(t_{\mathrm{sf}} \equiv \frac{1}{c} t_{\mathrm{dyn}}\right)$, where the star formation timescale parameter $c=0.1$ is adopted [8]. If a gas particle satisfies the above star formation criteria, a fractional part of the mass of the gas particle turns into a star particle. Since an individual star particle has a mass of $10^{5-7} M_{\odot}$, it dose not represent a single star, but an association of many stars. The mass of the stars associated with each star particle is distributed according to an initial mass function (IMF). We adopt a Salpter IMF with a slope $x=1.35$. iv) For the feedback of energy and heavy elements, we do not adopt the instantaneous recycling approximation. Via stellar winds, SNe II, and SNe Ia, thermal energy and heavy elements are ejected from an evolved star particle as functions of time and metallicity, and are distributed to all surrounding gas particles out to a constant radius of $1 \mathrm{kpc}(\S 5)$, or with the 

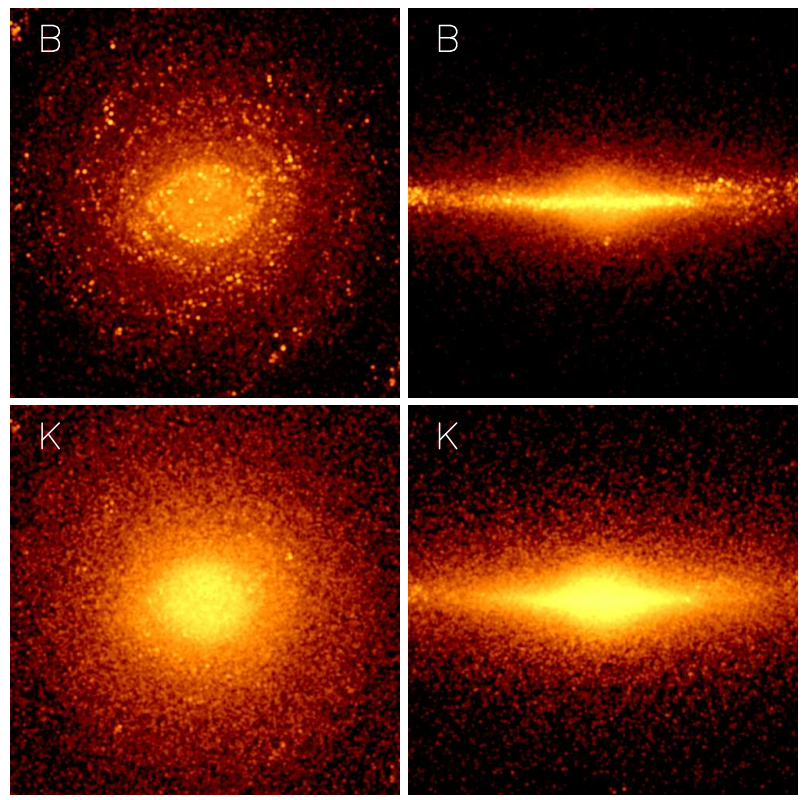

Figure 2: Luminosity Map of our simulated Milky Way-type galaxy at present for the rest B (upper panels) and $\mathrm{K}$ (lower panels) bands. Each panel is $20 \mathrm{kpc}$ on a side.

number of feedback neighbors $N_{\mathrm{FB}}=405$ (§6). v) The photometric evolution of a star particle is identical to the evolution of a simple stellar population (SSP). SSP spectra are taken from Kodama $\&$ Arimoto (1997) as a function of age and metallicity.

\section{Chemodynamical Evolution of the Milky Way Galaxy}

We simulate the chemodynamical evolution of the Milky Way-type galaxies from the CDM initial fluctuation. The initial condition is similar to those in Kobayashi $(2004,2005)$, but with the initial angular momentum of $\lambda \sim 0.1$, the total mass of $\sim 10^{12} M_{\odot}$, and $\sim 120000$ particles. We choose an initial condition where the galaxy does not undergo major mergers, otherwise no disk galaxy can form. The cosmological parameters are set to be $H_{0}=70 \mathrm{~km} \mathrm{~s}^{-1} \mathrm{Mpc}^{-1}, \Omega_{m}=0.3$, and $\Omega_{\Lambda}=0.7$.

In the CDM scenario, any galaxies form through the successive merging of subgalaxies with various masses. In our simulation, the merging of subgalaxies induces an initial starburst and a bulge forms by $z \gtrsim 3$. According to the late gas accretion, a disk structure is seen at $z \lesssim 2$. At present, the bulge and disk structures are well seen in K and B bands, respectively (Fig.2). 80\% of the bulge stars $(r \leq 1 \mathrm{kpc})$ are older than $\sim 10 \mathrm{Gyr}$. In the solar neighborhood $(r=7.5-8.5 \mathrm{kpc}$, $|z| \leq 0.5 \mathrm{kpc}), 50 \%$ of the disk stars are younger than $\sim 8 \mathrm{Gyr}$, and old stars tend to have small rotation velocity $v$ and large velocity dispersion $\sigma$. When we define thick disk as $v / \sigma<1.5,80 \%$ of thick disk stars are older than $\sim 8 \mathrm{Gyr}$.

The age-metallicity relations are shown in the upper panels of Figure 3. (a) In the solar neighborhood, $[\mathrm{Fe} / \mathrm{H}]$ increases to $\sim 0$ at $t \sim 2 \mathrm{Gyr}$, which is broadly consistent with the observation (Nordström et al. 2004). (c) In the thick disk, the relation is similar as in the solar neighborhood, 


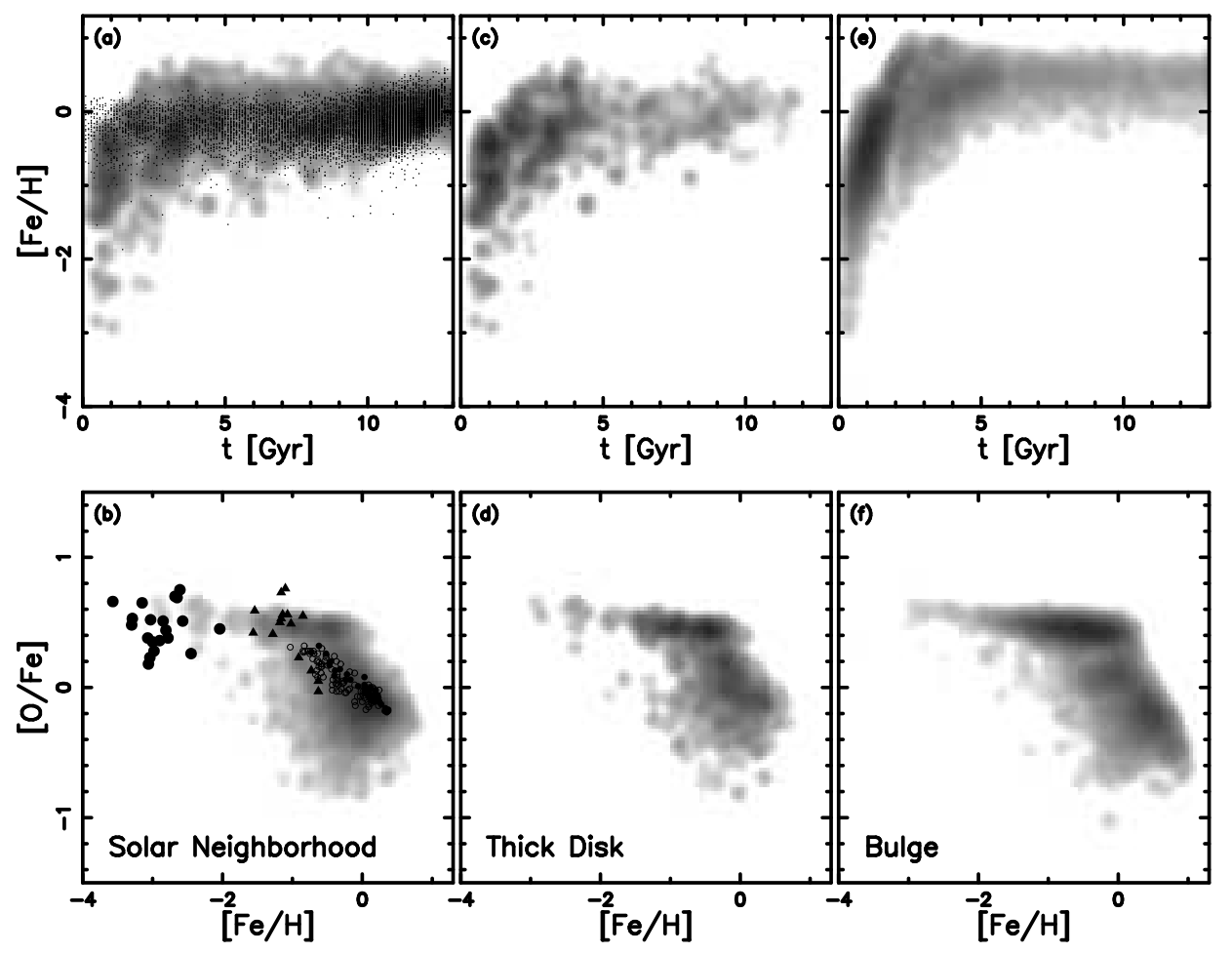

Figure 3: Age-metallicity relations (upper panels) and $[\mathrm{O} / \mathrm{Fe}]-[\mathrm{Fe} / \mathrm{H}]$ relations (lower panels) in the solar neighborhood ( $a$ and $b$ ), thick disk ( $c$ and d), and bulge (e and f). The contours show the mass density for the simulation. The dots show the observations of stars in the solar neighborhood (see the text for data sources).

but most stars are populated in the region with old age and low $[\mathrm{Fe} / \mathrm{H}]$. (e) In the bulge, $[\mathrm{Fe} / \mathrm{H}]$ increases more quickly than in the disks. Metal-rich stars with $[\mathrm{Fe} / \mathrm{H}] \sim 1$ appear at $t \sim 2 \mathrm{Gyr}$.

The $[\mathrm{O} / \mathrm{Fe}]-[\mathrm{Fe} / \mathrm{H}]$ relations are shown in the lower panels of Figure 3, and we obtain similar results for other $\alpha$-elements. (b) In the solar neighborhood, we can reproduce the observational trend (Edvardsson et al. 1993, open circles; Bensby et al. 2004, small filled circles; Gratton et al. 2003, triangles; Cayrel et al. 2004, large filled circles). [ $\alpha / \mathrm{Fe}]$ decreases because of the delayed iron enrichment of SNe Ia. If we do not include the metallicity effect on SNe Ia, or if we do not include $\mathrm{HNe}$, we cannot reproduce the plateau at $[\mathrm{Fe} / \mathrm{H}] \lesssim-1$, and the scatter of $[\alpha / \mathrm{Fe}]$ at $[\mathrm{Fe} / \mathrm{H}]$ $\lesssim-1$ is too large. At $[\mathrm{Fe} / \mathrm{H}] \gtrsim-1$, the scatter is large. This may be because the mixing of heavy elements among gas particles has not been included in our chemodynamical model.

(d) In the thick disk, chemical enrichment timescale is so short that $[\alpha / \mathrm{Fe}]$ tends to be larger than in the thin disk, which is consistent with the observations (Bensby et al. 2004). (f) In the bulge, chemical enrichment timescale is shorter than in the disks, the $[\alpha / \mathrm{Fe}]$ plateau continues to $[\mathrm{Fe} / \mathrm{H}] \sim 0$, which is consistent with some observations (Zoccali et al. 2008). The star formation has not been terminated in the simulation, and some new stars are forming also in the bulge. Such young stars tend to have large $[\mathrm{Fe} / \mathrm{H}]$ and low $[\alpha / \mathrm{Fe}]$ in our simulation, and the observed stars in Cunha et al. (2007) may be affected by inhomogeneity, or some uninvolved physics. Particularly, if the relations of $\mathrm{O}$ and $\mathrm{Mg}$ are different, we may have to include non-supernova physics such as strong stellar winds. 

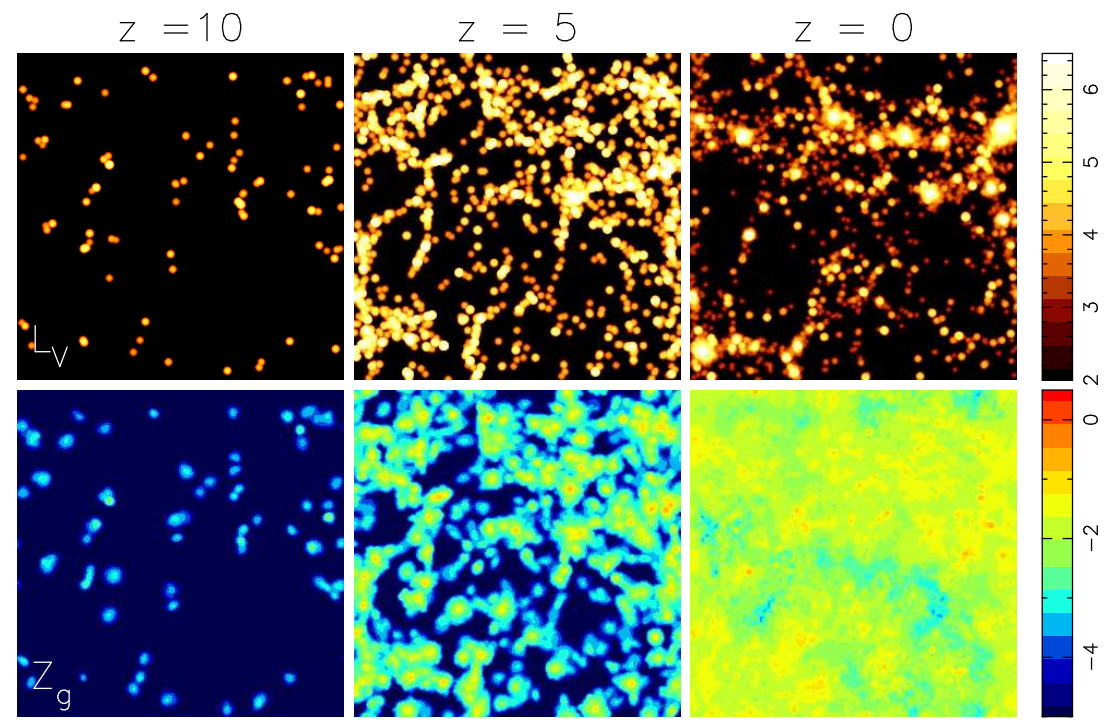

Figure 4: The time evolution of our cosmological simulation in a periodic box $10 h^{-1} \mathrm{Mpc}$ on a side. We show the projected stellar V-luminosity (upper panels) and gas metallicity $\log Z_{\mathrm{g}} / Z_{\odot}$ (lower panels).

We also trace the orbit of star particles, and study the origin of the thick disk. The fractions of stars that have formed in the disk $(z<1 \mathrm{kpc})$ are $\sim 40 \%$. The rest, more than half of thick disk stars have formed in merging subgalaxies before they accrete onto the disk. In this sense, the CDM picture seems not to conflict with the Milky Way Galaxy. However, it is very hard to find initial condition to form disk galaxies (with this resolution) because major mergers brake the disk structure, and late star formation caused by slow gas accretion is not enough to re-generate (contrary to Steinmetz \& Navarro 2002). For the number of disk galaxies, the CDM picture seems to have a problem, although it should be tested with cosmological simulations.

We succeed in reproducing the kinematical and chemical properties of the Milky Way Galaxy, with adopting Salpeter IMF for all component. Pair-instability supernovae, which produce much more $\mathrm{Fe}$, more $[\mathrm{S} / \mathrm{Fe}]$, and less $[\mathrm{Zn} / \mathrm{Fe}]$, seem not to contribute the chemical evolution of Milky way Galaxy. From statistical comparison for the frequency distribution in the $[\mathrm{X} / \mathrm{Fe}]-[\mathrm{Fe} / \mathrm{H}]$ diagrams, chemodynamical models should be tested and improved. Different components of the Milky Way Galaxy have different stellar populations, namely, different elemental abundance patterns. Using the future observations of abundance ratios, we can know when and where the stars form. The galactic archaeology can be a powerful subject with chemodynamical simulations.

\section{Cosmological Simulations}

We simulate the evolution of dark matter, gas, and stellar systems and the chemical enrichment from SNe II, SNe Ia, and HNe, from the cosmological initial condition with $H_{0}=70 \mathrm{~km} \mathrm{~s}^{-1}$ $\mathrm{Mpc}^{-1}, \Omega_{m}=0.3, \Omega_{\Lambda}=0.7, \Omega_{\mathrm{b}}=0.04, n=1$, and $\sigma_{8}=0.9$. The initial condition is calculated in a $10 h^{-1} \mathrm{Mpc}$ cubic box with periodic boundary conditions with $N_{\mathrm{DM}}=N_{\text {gas }}=96^{3}$ [1]. Figure 4 shows the time evolution of the densities of gas, stellar V-band luminosity and gas metallicity for the high resolution simulation. Star formation takes place in a distributed fashion at high redshifts, 

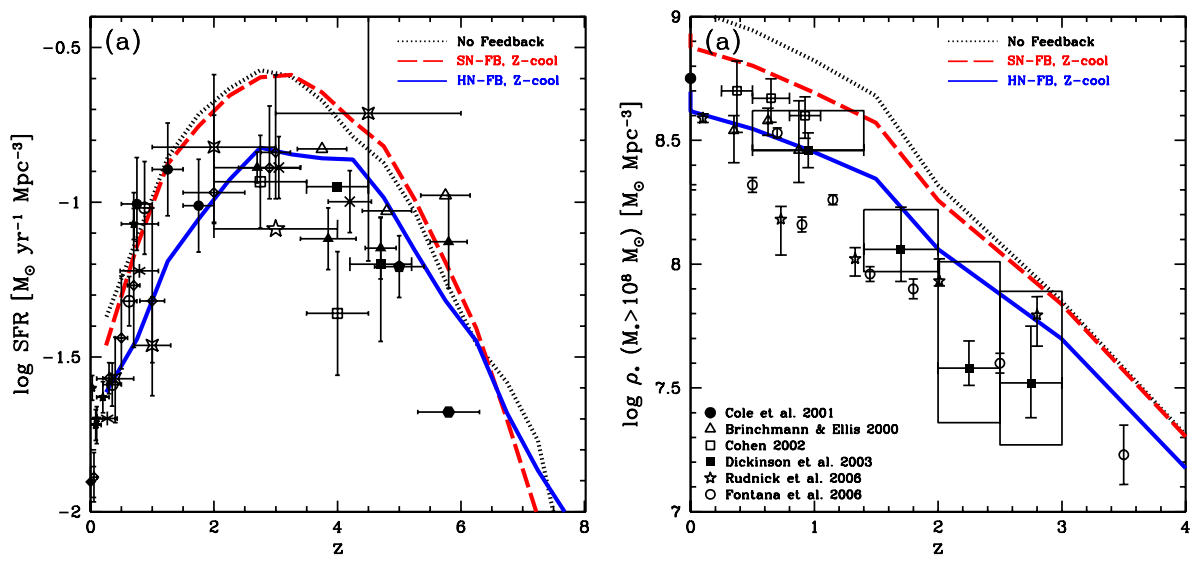

Figure 5: (a) Cosmic star formation rates for no feedback case (dotted line) and for the SN (dashed line) and HN (solid line) feedback with the metal-dependent cooling. See K07 for the references of rest-frame U-band, IR, and $\mathrm{H} \alpha$ observations. Rest-frame UV observations are plotted with dust correction (Steidel et al. 1999). (b) Redshift evolution of iron abundances of gas. The points show individual galaxies with the size representing the size of galaxies. The solid, dashed, and dotted lines are for the mean, the largest galaxy in the simulated region, and the IGM, respectively. See the text for the observational data (errorbars, box).

and becomes most active around $z \sim 4$. The distribution of stars appears smooth at high redshifts, but concentrated at lower redshifts. Because of the feedback, massive galaxies are surrounded by hot gas, and heavy elements are also distributed in the intergalactic medium. Both in the gas phase and stars, metallicity gradients are generated in high density regions. At present, $\sim 7000$ galaxies are identified with the friend-of-friends group-finding algorithm in this simulated field.

Figure 5a shows the cosmic star formation rates (SFRs) that are directly measured from the ages of stellar particles for the low resolution simulations. Supernova (SN) feedback (dashed line) decreases the SFR from $z \sim 3$, but metal-dependent cooling increases as large as no feedback case (dotted line). If we include hypernova (HN) feedback (solid line), the SFR starts to be suppressed from $z \sim 6$ onwards, and is overall smaller by a factor of 3 at $0 \lesssim z<3$. The resulting SFR is in broad agreement with the observations that show a peak of $\log \left(\mathrm{SFR} /\left[\mathrm{M}_{\odot} \mathrm{yr}^{-1}\right]\right) \sim-1$ at $z \sim 3$.

Without feedback, $25 \%$ of baryons turn into stars, which is too large. With SN feedback, the stellar fraction reduces to $10-15 \%$. The larger energy ejection by HNe results in the stellar fraction less than $10 \%$, which is consistent with the recent observational estimate (Fukugita \& Peebles 2004). The present mean gas metallicity is $[\mathrm{Fe} / \mathrm{H}] \sim-1.3$ for $\mathrm{SN}$ feedback, and is reduced to $\sim-1.8$ for HN feedback. The mean stellar metallicity is almost solar for the SN feedback, and becomes sub-solar for the HN feedback.

The metal enrichment timescale depends on the environment. Figure $5 \mathrm{~b}$ shows the evolution of oxygen abundances in the gas phase in different environments. In large galaxies, enrichment takes place so quickly that $[\mathrm{O} / \mathrm{H}]$ reaches $\sim-1$ at $z \sim 7$, which is consistent with the sub-solar metallicities of the Lyman break galaxies (large errorbar at $z=3$, Pettini et al. 2001). The low metallicities of the damped Lyman $\alpha$ systems (errorbars, Prochaska et al. 2003) are also consistent with our galaxies, provided these systems are dwarf galaxies or the outskirts of massive galaxies. The low $[\mathrm{C} / \mathrm{H}]$ of the intergalactic medium (IGM) (box, Schaye et al. 2003) can be explained if the 

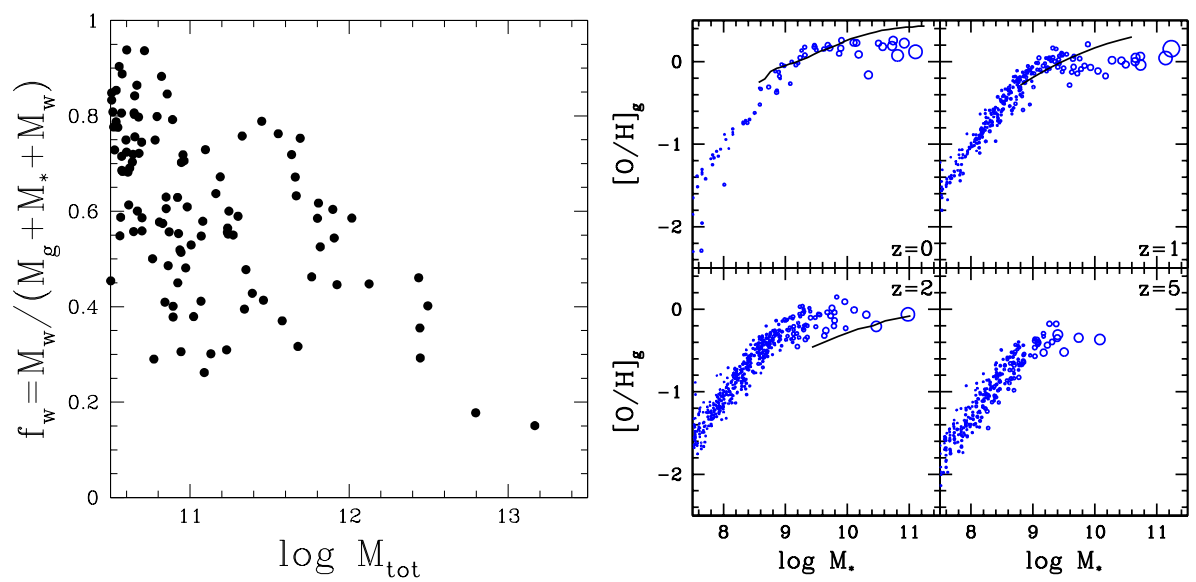

Figure 6: (a) The wind fraction $f_{\mathrm{w}}=M_{\mathrm{w}} /\left(M_{\mathrm{g}}+M_{*}+M_{\mathrm{w}}\right)$ against the total mass. (b) Mean metallicities of cold gas $\left(T<10^{4} \mathrm{~K}\right)$ within $10 \mathrm{kpc}$, plotted against the total stellar mass at $z=0,1,2$, and 5 . See the text for the observational data (solid lines).

IGM is enriched only by SNe II and HNe. The average metallicity of the universe reaches $[\mathrm{O} / \mathrm{H}]$ $\sim-2$ and $[\mathrm{Fe} / \mathrm{H}] \sim-2.5$ at $z \sim 4$, but reaches the same values at $z \sim 3$ in the IGM.

When and where do stars form? To answer this question, we break up the cosmic SFR history according to galaxy mass (see Figs. 13 and 14 in K07). As a result, the majority of the stars in present-day massive galaxies have formed in smaller systems at high redshifts, and the stellar population of these galaxies is as old as $10 \mathrm{Gyr}$, consistent with observations of elliptical galaxies. No relation is found between luminosity-weighted age and stellar mass. The ages of low-mass galaxies span a wide range of $1-10 \mathrm{Gyr}$.

How are heavy elements ejected from galaxies to the IGM? In the simulation, we can trace the orbit of gas particles over time. Exploiting this, we define as wind particles those that are not in galaxies now, but have been in galaxies before (see Fig. 15 in K07). In this simulation, $\sim 10 \%$ of baryons turn into stars, $\sim 10 \%$ of the gas stays in galaxies ( $\sim 8 \%$ is hot), and $\sim 20 \%$ is ejected as galactic winds. The rest, half of the baryons, never accretes onto galaxies.

When we follow the orbits of gas particles, we can also examine from which galaxies the wind gas particles are ejected. This allows a measurement of the ejected wind mass from each galaxy. In Figure $6 \mathrm{a}$, we plot the wind fraction, i.e. the ratio between the total wind mass to the total accreted baryon mass, against the total mass. A clear relation is found between the wind fraction and the total mass. Winds are efficiently ejected from small galaxies, with $\sim 80 \%$ of accreted baryons being ejected from $M_{\mathrm{tot}} \sim 10^{11} \mathrm{M}_{\odot}$ galaxies. A similar relation is also found for the ejected metal fraction, i.e. the ratio between the wind metal mass to the total metal mass. It is interesting that the wind fraction and the ejected metal fraction correlate well with the stellar metallicity. Based on this finding, we conclude that the origin of the mass-metallicity relation can be explained with the galactic winds.

In galaxies, metallicity of the cold gas increases with galaxy mass (Fig.6b), which is comparable to observations with a large scatter (solid line at $z=0$, Tremonti et al. 2004; $z=2$, Erb et al. 2006). The central cold gas shows a relation between galaxy mass and metallicity with 


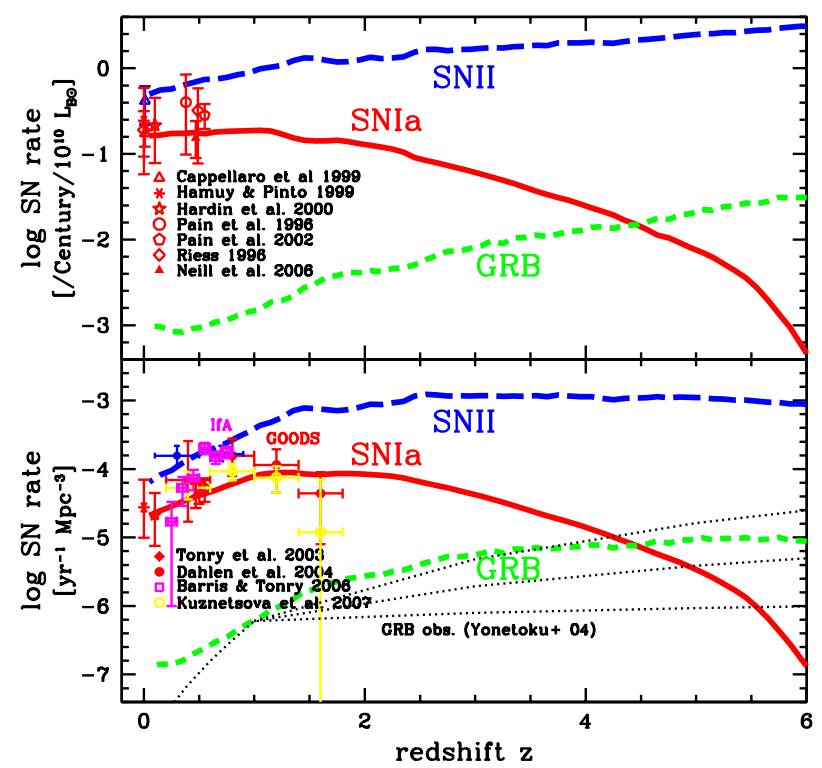

Figure 7: The cosmic rates for SNe II (long-dashed line), SNe Ia (solid line), and GRBs (short-dashed line) per luminosity ( $\mathrm{SNu}$, upper panel) and volume (lower-panel). The dotted line shows the observational estimate of GRB rates (Yonetoku et al. 2004). The open triangle and small filled circle are for the observed SN II rates, and the other symbols are for the SN Ia rates. See [6] for the observational data sources.

shallower slope than observed in emission-line galaxies. For the stellar population, the observed mass-metallicity relation is well reproduced (Kobayashi \& Arimoto 1999; Pahre et al. 1998; Gallazzi et al. 2005), and originates in mass-dependent galactic winds. These relations are present since $z \sim 5$.

In our simulations, the effects of hypernovae are included, and we can also predict the cosmic gamma-ray burst (GRB) rate history, assuming that the progenitors of GRB are massive $\left(\geq 40 M_{\odot}\right)$ hypernovae. Since the progenitors of GRBs are likely to be fast rotating, and thus metallicity should be low to avoid mass loss. Figure 7, the hypernova efficiency is set to be 0.5 for $Z \leq 0.001$ and decreases to 0.01 for $Z=Z_{\odot}$. The predicted GRB rate history is in good agreement with the observational estimates by Yonetoku et al. (2004). The present hypernova rate is consistent with the observational estimate by Podsiadlowski et al. (2004). The SN II rate simply traces the SFR history, and peaks at $z \sim 3$, while the SN Ia rate slightly increases toward $z \sim 2$, then rapidly decreases, because of the both lifetime and metallicity effects.

\section{References}

[1] Kobayashi, C., Springel, V, \& White, S. D. M. 2007, MNRAS, 376, 1465 (K07)

[2] Nomoto, K., Maeda, K., Umeda, H., Ohkubo, T., Deng, J., \& Mazzali, P. 2003, in IAU Symp. 212, 395

[3] Kobayashi, C., Umeda, H., Nomoto, K., Tominaga, N., \& Ohkubo, T. 2006, ApJ, 653, 1145 (K06)

[4] Kobayashi, C., Tsujimoto, T., Nomoto, K., Hachisu, I, \& Kato, M. 1998, ApJ, 503, L155

[5] Hachisu, I., Kato, M., \& Nomoto, K. 2008, ApJ, 679, 1390

[6] Kobayashi, C. \& Nomoto, K. 2008, ApJ, submitted, astro-ph/0801.0215

[7] Kobayashi, C., 2004, MNRAS, 347, 740

[8] Kobayashi, C., 2005, MNRAS, 361, 1216 